\title{
A Generalized Monte Carlo Approach for the Analysis of Quantum-Transport Phenomena in Mesoscopic Systems: Interplay Between Coherence and Relaxation
}

\author{
FAUSTO ROSSI $^{\mathrm{a}, *}$ STEFANO RAGAZZI $^{\mathrm{b}}$, ALDO DI CARLO $^{\mathrm{b}}$ and PAOLO LUGLI ${ }^{\mathrm{b}}$ \\ a Istituto Nazionale Fisica della Materia (INFM) and Dipartimento di Fisica, Universitá di Modena, I-41100 Modena, Italy; \\ b INFM and Dipartimento di Ingegneria Elettronica, Università di Roma "Tor Vergata", I-00133 Roma, Italy
}

\begin{abstract}
A theoretical investigation of quantum-transport phenomena in mesoscopic systems is presented. In particular, a generalization to "open systems" of the well-known Semiconductor Bloch equations is proposed. Compared to the conventional Bloch theory, the presence of spatial boundary conditions manifest itself through self-energy corrections and additional source terms in the kinetic equations, which are solved by means of a generalized Monte Carlo simulation. The proposed numerical approach is applied to the study of the scattering-induced suppression of Bloch oscillations in semiconductor superlattices as well as to the analysis of quantum-transport phenomena in double-barrier structures.
\end{abstract}

Keywords: Quantum, transport, simulation, mesoscopic, coherence, dissipation

\section{INTRODUCTION}

The Monte Carlo (MC) method, which has been applied for more than 25 years for calculation of semiclassical charge transport in semiconductors, is the most powerful numerical tool for microelectronics device simulation [1]. However, it is well known that present-day technology pushes device dimensions toward limits where the traditional semiclassical transport theory can no longer be applied and a more rigorous quantum transport theory is required [2].
In this paper, a generalized MC approach for the analysis of hot-carrier transport and relaxation phenomena in quantum devices is proposed. The method is based on a generalized MC solution of the set of kinetic equations governing the time evolution of the single-particle density matrix. Our approach can be regarded as an extension to open systems of the generalized MC method recently proposed for the analysis of the coupled coherent and incoherent carrier dynamics in photoexcited semiconductors [3]. Compared to more academic quantum-kinetic approaches [4] - whose applica-

\footnotetext{
* Corresponding author.
} 
tion is often limited to highly simplified physical models and conditions - , the proposed simulation scheme can be applied to realistic cases, allowing on the one hand a proper description of quantuminterference phenomena induced by the potential profile and on the other hand maintaining all the well known advantages of the conventional MC method.

As a first application, we will discuss the scattering-induced suppression of Bloch oscillations in semiconductor superlattices. As a second example, we will study the strong interplay between coherence and relaxation within a double-barrier structure.

\section{THEORETICAL APPROACH}

In order to properly describe carrier-transport phenomena in mesoscopic structures, let us consider a generic electron-phonon system, whose Hamiltonian can be schematically written as:

$$
\boldsymbol{H}=\left(\boldsymbol{H}_{c}+\boldsymbol{H}_{p}\right)+\left(\boldsymbol{H}_{c c}+\boldsymbol{H}_{c p}\right)=\boldsymbol{H}_{0}+\boldsymbol{H}^{\prime} .
$$

The single-particle term $\boldsymbol{H}_{0}$ is the sum of the noninteracting carrier and phonon Hamiltonians $\boldsymbol{H}_{c}$ and $\boldsymbol{H}_{p}$ while the many-body contribution $\boldsymbol{H}^{\prime}$ includes carrier-carrier as well as carrier-phonon interactions. More specifically, the single-particle Hamiltonian $\boldsymbol{H}_{c}$ describes the noninteracting carrier system within the potential profile of our mesoscopic structure (including possible external fields). By denoting with $\phi_{\alpha}(\boldsymbol{r})=\langle\boldsymbol{r} \mid \alpha\rangle$ the wavefunction of the single-particle state $\alpha$ and with $\varepsilon_{\alpha}$ the corresponding energy, the noninteractingcarrier Hamiltonian reads:

$$
\boldsymbol{H}_{c}=\sum_{\alpha} \varepsilon_{\alpha} a_{\alpha}^{\dagger} a_{\alpha}
$$

Here, the usual second-quantization picture in terms of creation $\left(a_{\alpha}^{\dagger}\right)$ and destruction $\left(a_{\alpha}\right)$ operators has been employed.
The basic quantity in our theoretical approach is the single-particle density matrix

$$
\rho_{\alpha \beta}=\left\langle a_{\beta}^{\dagger} a_{\alpha}\right\rangle
$$

Its diagonal elements correspond to the usual distribution functions of the semiclassical Boltzmann theory $\left(f_{\alpha}=\rho_{\alpha \alpha}\right)$ while the off-diagonal terms $(\alpha \neq \beta)$ describe the degree of quantummechanical phase coherence between states $\alpha$ and $\beta$. Starting from the Heisenberg equations of motion for the operators $a_{\alpha}$ it is possible to derive a set of equations of motion for the density-matrix elements $\rho_{\alpha \beta}$, called semiconductor Bloch equations (SBE) [5], whose general structure is given by:

$$
\frac{d}{d t} \rho_{\alpha \beta}=\left.\frac{d}{d t} \rho_{\alpha \beta}\right|_{\boldsymbol{H}_{0}}+\left.\frac{d}{d t} \rho_{\alpha \beta}\right|_{\boldsymbol{H}^{\prime}} .
$$

The time evolution induced by the single-particle Hamiltonian $\boldsymbol{H}_{0}$ can be evaluated exactly yielding:

$$
\left.\frac{d}{d t} \rho_{\alpha \beta}\right|_{\boldsymbol{H}_{0}}=\frac{1}{i \hbar} \sum_{\alpha^{\prime} \beta^{\prime}} \mathcal{E}_{\alpha \beta, \alpha^{\prime} \beta^{\prime}} \rho_{\alpha^{\prime} \beta^{\prime}}=-i \omega_{\alpha \beta} \rho_{\alpha \beta} .
$$

with $\omega_{\alpha \beta}=\left(\varepsilon_{\alpha}-\varepsilon_{\beta}\right) / \hbar$. On the contrary, the time evolution due to the many-body Hamiltonian $\boldsymbol{H}^{\prime}$ involves phonon-assisted as well as higher-order density-matrices; Thus, approximations are needed in order to "close" our set of equations of motion (with respect to our kinetic variables). In particular, the "mean-field" approximation together with the Markov limit approximation allow us to derive a set of closed equations of motion which is still local in time:

$$
\left.\frac{d}{d t} \rho_{\alpha \beta}\right|_{\boldsymbol{H}^{\prime}}=\sum_{\alpha^{\prime} \beta^{\prime}} \Gamma_{\alpha \beta, \alpha^{\prime} \beta^{\prime}}^{\prime} \rho_{\alpha^{\prime} \beta^{\prime}}
$$

The matrix $\Gamma_{\alpha \beta, \alpha^{\prime} \beta^{\prime}}^{\prime}$ is, in general, a complex quantity: its real part describes a sort of generalized scattering rate while its imaginary part is related to energy-renormalization phenomena. Recently, a full quantum-mechanical approach 
has been proposed [6], which overcomes the usual Markov limit in describing the carrier-phonon interaction. However, due to the huge amount of CPU time required, its applicability is still limited to short time-scales and extremely simplified situations.

The analysis presented so far is typical of a socalled "closed" system, i.e., a physical system defined over the whole coordinate space. However, this is not the case of interest for the analysis of quantum-transport phenomena in mesoscopic devices, where the properties of the carrier subsystem are strongly influenced by spatial boundary conditions. In order to better understand this crucial point, let us reconsider our theoretical scheme in terms of real-space coordinates.The proper quantum-mechanical description is then based on the so-called Wigner function [7], given by the following Weyl-Wigner transformation of the single-particle density matrix:

$$
f^{W}(\boldsymbol{r}, \boldsymbol{k})=\sum_{\alpha \beta} \rho_{\alpha \beta} u_{\alpha \beta}(\boldsymbol{r}, \boldsymbol{k})
$$

with

$$
\begin{aligned}
u_{\alpha \beta}(\boldsymbol{r}, \boldsymbol{k})= & (2 \pi)^{-\frac{3}{2}} \int d \boldsymbol{r}^{\prime} \phi_{\alpha}\left(\boldsymbol{r}+\frac{1}{2} \boldsymbol{r}^{\prime}\right) e^{-i \boldsymbol{k} \cdot \boldsymbol{r}^{\prime}} \times \\
& \times \phi_{\beta}^{*}\left(\boldsymbol{r}-\frac{1}{2} \boldsymbol{r}^{\prime}\right) .
\end{aligned}
$$

By applying the above Weyl-Wigner transformation to the non-interacting SBE (5), we obtain:

$$
\begin{aligned}
\left.\frac{d}{d t} f^{W}(\boldsymbol{r}, \boldsymbol{k}, t)\right|_{\boldsymbol{H}_{0}}= & \int d \boldsymbol{r}^{\prime} d \boldsymbol{k}^{\prime} L_{0}\left(\boldsymbol{r}, \boldsymbol{k} ; \boldsymbol{r}^{\prime}, \boldsymbol{k}^{\prime}\right) \times \\
& \times f^{W}\left(\boldsymbol{r}^{\prime}, \boldsymbol{k}^{\prime}, t\right)
\end{aligned}
$$

where

$$
L_{0}\left(\boldsymbol{r}, \boldsymbol{k} ; \boldsymbol{r}^{\prime}, \boldsymbol{k}^{\prime}\right)=-i \sum_{\alpha \beta} u_{\alpha \beta}(\boldsymbol{r}, \boldsymbol{k}) \omega_{\alpha \beta} u_{\alpha \beta}^{*}\left(\boldsymbol{r}^{\prime}, \boldsymbol{k}^{\prime}\right)
$$

is the single-particle Liouville operator in the $\boldsymbol{r}, \boldsymbol{k}$ representation.
For the case of a closed system, the Wigner function $f^{W}$ is defined for any value of the realspace coordinate $\boldsymbol{r}$ and its time evolution is fully determined by its initial condition.

On the contrary, for the case of an open system, $f^{W}$ is defined only within a given region $\Omega$ of interest and its time evolution is determined by the initial condition $f_{0}^{W}$ inside such region as well as by its values on the boundary $r_{b}$ of the domain $\Omega$ at any time $t^{\prime}>t_{0}$. More specifically, by applying the Green's function theory [8] to Eq. (9) we have:

$$
\begin{aligned}
& f^{W}(\boldsymbol{r}, \boldsymbol{k} ; t)= \int_{\Omega} d \boldsymbol{r}^{\prime} \int d \boldsymbol{k}^{\prime} G_{0}\left(\boldsymbol{r}, \boldsymbol{k} ; \boldsymbol{r}^{\prime}, \boldsymbol{k}^{\prime} ; t-t_{0}\right) \times \\
& \times f^{W}\left(\boldsymbol{r}^{\prime}, \boldsymbol{k}^{\prime} ; t_{0}\right)+ \\
&+\int d \boldsymbol{r}_{b} \int d \boldsymbol{k}^{\prime} \int_{t_{0}}^{t} d t^{\prime} G_{0}\left(\boldsymbol{r}, \boldsymbol{k} ; \boldsymbol{r}_{b}, \boldsymbol{k}^{\prime} ; t-t^{\prime}\right) \times \\
& \quad \times f_{b}^{W}\left(\boldsymbol{r}_{b}, \boldsymbol{k}^{\prime}, t^{\prime}\right) v\left(\boldsymbol{k}^{\prime}\right),
\end{aligned}
$$

where

$$
G_{0}\left(\boldsymbol{r}, \boldsymbol{k} ; \boldsymbol{r}^{\prime}, \boldsymbol{k}^{\prime} ; \tau\right)=\sum_{\alpha \beta} u_{\alpha \beta}(\boldsymbol{r}, \boldsymbol{k}) e^{-i \omega_{\alpha \beta} \tau} u_{\alpha \beta}^{*}\left(\boldsymbol{r}^{\prime} \boldsymbol{k}^{\prime}\right)
$$

is the single-particle evolution operator, while $v(\boldsymbol{k})$ is the component of the carrier group velocity normal to the boundary surface. We clearly see that the value of $f^{W}$ is obtained from the single-particle propagation of the initial condition $f_{0}^{W}$ inside the domain $\Omega$ plus the propagation of the boundary values $f_{b}^{W}$ from the points of the surface at any time $t^{\prime}$ to the point $\boldsymbol{r}, \boldsymbol{k}$ of interest.

Let us now come back to the density-matrix formulation by introducing the inverse WeylWigner transform:

$$
\bar{\rho}_{\alpha \beta}=\int_{\Omega} d \boldsymbol{r} \int d \boldsymbol{k} u_{\alpha \beta}^{*}(\boldsymbol{r}, \boldsymbol{k}) f^{W}(\boldsymbol{r}, \boldsymbol{k}) .
$$

The above density matrix $\bar{\rho}$ provides a description of our open system equivalent to the Wignerfunction one. By applying the above transforma- 
tion to Eq. (11) and then performing its time derivative, we finally obtain:

$$
\left.\frac{d}{d t} \bar{\rho}_{\alpha \beta}\right|_{H_{0}}=\frac{1}{i \hbar} \sum_{\alpha^{\prime} \beta^{\prime}} \overline{\mathcal{E}}_{\alpha \beta, \alpha^{\prime} \beta^{\prime}} \bar{\rho}_{\alpha^{\prime} \beta^{\prime}}+\bar{S}_{\alpha \beta},
$$

where

$$
\overline{\mathcal{E}}_{\alpha \beta, \alpha^{\prime} \beta^{\prime}}=\left[U \mathcal{E} U^{-1}\right]_{\alpha \beta, \alpha^{\prime} \beta^{\prime}}
$$

is the single-particle self-energy tensor in Eq. (5) "dressed" by the transformation

$$
U_{\alpha \beta, \alpha^{\prime} \beta^{\prime}}=\int_{\Omega} d \boldsymbol{r} \int d \boldsymbol{k} u_{\alpha \beta}^{*}(\boldsymbol{r}, \boldsymbol{k}) u_{\alpha^{\prime} \beta^{\prime}}(\boldsymbol{r}, \boldsymbol{k}),
$$

while

$$
\begin{aligned}
\bar{S}_{\alpha \beta}= & \sum_{\alpha^{\prime} \beta^{\prime}} U_{\alpha \beta, \alpha^{\prime} \beta^{\prime}} \int d \boldsymbol{r}_{b} \int d \boldsymbol{k} u_{\alpha^{\prime} \beta^{\prime}}^{*}\left(\boldsymbol{r}_{b}, \boldsymbol{k}\right) v(\boldsymbol{k}) \times \\
& \times f_{b}^{W}\left(\boldsymbol{r}_{b}, \boldsymbol{k}\right)
\end{aligned}
$$

is a source term induced by our spatial boundary conditions.

Eq. (14) is the desired generalization to the case of open systems of the standard single-particle SBE in Eq. (5). In addition to the source term (17) (whose explicit form depends on the particular values of $f_{b}^{W}$ on the surface of our domain), the presence of spatial boundary conditions induces modifications on the self energy $\mathcal{E}$ of the system via the transformation $U$ in Eq. (16).

\section{SOME SIMULATED EXPERIMENTS}

The theoretical approach presented so far is the starting point of our MC simulation. The generalized SBE (4) are solved by means of the same MC simulation scheme described in [3]. The method is based on a time-step separation between coherent and incoherent dynamics (see Eq. (4)). The former accounts in a rigorous way for all quantum phenomena induced by the potential profile of the device as well as for the proper boundary conditions (see Eq. (14)). The latter, described within the basis given by the eigenstates $\alpha$ of the potential profile (see Eq. (6)), accounts for all the relevant scattering mechanisms by means of a conventional "ensemble" MC simulation [1].

The above numerical approach has been applied to the study of the scattering-induced suppression of Bloch oscillations in semiconductor superlattices and to the analysis of quantum-transport phenomena in a double-barrier structure.

In the first case, a biased GaAs/AlGaAs multiquantum-well (MQW) has been considered (15 periods of a $20 \AA$ barrier and a $100 \AA$ well with a barrier height of $0.5 \mathrm{eV}$ ). The electric field in the MQW region is about $12 \mathrm{kV} / \mathrm{cm}$. The ultrafast dynamics of an injected wavepacket (injection energy $E=50 \mathrm{meV}$ ) has been simulated. Figure 1 shows the position mean value of the wavepacket as a function of time. For the scattering-free case, the expected Bloch oscillations in real space are well reproduced. They originate from the coherent motion of our wavepacket through the superlattice structure. In the presence of scattering mechanisms, such oscillations are strongly damped. This is mainly due to carrier-LO phonon scattering, whose typical time-scale is comparable with the

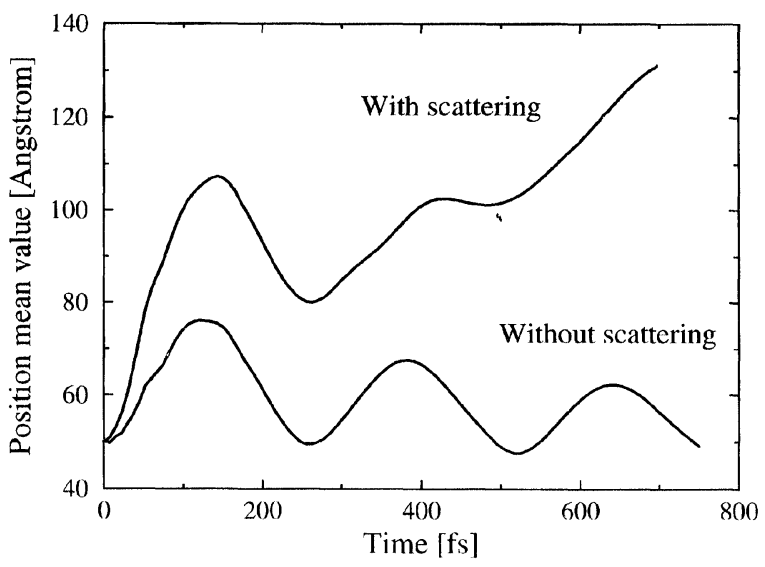

FIGURE 1 Position mean value of an electronic wavepacket within a biased semiconductor super lattice as a function of time with and without scattering (see text). 
sub-picosecond time-scale of our Bloch oscillations.

We have than carried out the simulation of an electronic wavepacket entering (from left) a double-barrier structure. The injected carriers are energetically in resonance with the second level of the well. In Figure 2 the charge distribution across the double-barrier structure is shown at different times with and without scattering. As expected, for the scattering-free case (Fig. 2b) we obtain the well-established resonance scenario: the wavepacket enters in resonance with the second energy level of the structure and after some time-delay a part of it is transmitted and a part is reflected. On the contrary, in the presence of scattering events (Fig. $2 \mathrm{a}$ ), the resonance process is strongly influenced by a charge transfer from the second to the first energy level. This effect is clearly evidenced by the charge distribution inside the well region, which for the free-scattering case exhibits the typical
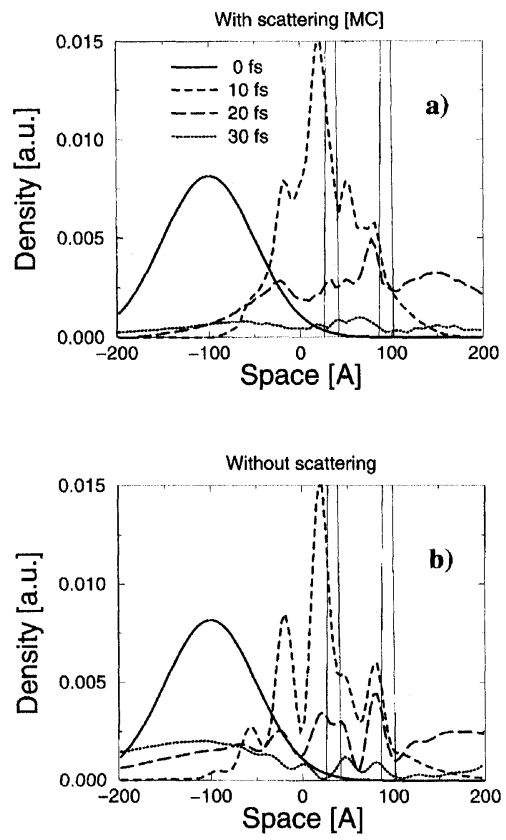

FIGURE 2 Charge distribution across a double-barrier structure at different times corresponding to an electron wavepacket injected in resonance with the second level (see text). The scattering rates have been artificially increased by a factor 10 with respect to the GaAs values in order to emphasize the relaxation to the first level. second-level symmetry while in the presence of scattering it approaches the symmetry of the ground level.

\section{CONCLUSIONS}

In this paper, we have presented a generalization to open systems of the well-known semiconductor Bloch equations. The proposed approach, based on the density-matrix formalism, allows a proper description of the strong coupling between coherent and incoherent dynamics in mesoscopic systems. These generalized SBE are numerically solved by means of the same MC simulation scheme used already for the study of ultrafast carrier dynamics in photoexcited semiconductors [3]. Our simulated experiments clearly show the failure of any pure coherent or incoherent approach in describing such quantum-transport regime.

\section{Acknowledgements}

We are grateful to Carlo Jacoboni, Tilmann Kuhn and Elisa Molinari for stimulating and fruitful discussions. This work was supported in part by the EC Commission through the Network "ULTRAFAST". One of us (ADC) acknowledges TRACS support.

\section{References}

[1] Jacoboni, C. and Lugli, P. (1989). The Monte Carlo Method for Semiconductor Device Simulations (Springer, Wien).

[2] Rossi, F., Brunetti, R. and Jacoboni, C. (1992). in Hot Carriers in Semiconductor Nanostructures: Physics and Applications, edited by J. Shah (Academic Press Inc., Boston), p. 153.

[3] Kuhn, T. and Rossi, F. (1992). Phys. Rev. Lett., 69, 977; Rossi, F., Haas, S. and Kuhn, T. (1994). Phys. Rev. Lett., 72, 152; Haas, S., Rossi, F. and Kuhn, T. (1996). Phys. Rev. $B, 53,12855$.

[4] Mclennan, M. J., Lee, Y. and Datta, S. (1991). Phys. Rev. $B, 43,13846$; Kluksdahl, N. C., Kriman, A. M., Ferry, D. K., Ringhofer, C. (1989). Phys. Rev. B, 39, 7720; Biegel, B. A. et al. (1996). Phys. Rev. B, 54, 8070.

[5] Haug, H. and Koch, S. W. (1994). Quantum Theory of the Optical and Electronic Properties of Semiconductors, 3rd Edn. (World Scientific, Singapore). 
[6] Brunetti, R., Jacoboni, C. and Price, P. J. (1994). Phys. Rev. B, 50, 11872

[7] Frensley, W. (1990). Rev. Mod. Phys., 62, 3.

[8] Morse, P. M. and Feshbach, H. (1953). Methods of theoretical physics, New York: McGraw-Hill.

\section{Author's Biographies}

Fausto Rossi born in 1962 in Carpi (Italy), Ph.D. in Physics in 1993 from Modena University. He is currently part of the INFM research unit of the Modena University. His research activity is mainly devoted to the analysis of non-equilibrium phenomena in semiconductors, namely ultrafast dynamics of photoexcited carriers and quantumtransport simulation.

Stefano Ragazzi graduated in Electronic Engineerings in 1997 at the El. Eng. Dept. on the University of Rome "Tor Vergata", he is currently enrolled in the Italian Navy.
Aldo Di Carlo graduated in Physics at the University of Roma, Italy, in 1991, obtained the Ph.D. degree in Physics in 1995 at the Walter Schottky Institute of the Technical University of Munich (Germany). He is currently researcher at the El. Eng. Dept. of the University of Roma, "Tor Vergata", where his work is mainly focused on the theoretical study of optical and transport processes in semiconductor nanostructures, devices and polymers.

Paolo Lugli born in Carpi (Italy) in 1956, obtained his Laurea in Physics at the University of Modena in 1979 and his Ph.D. in El. Eg. at Colorado State University in 1985. He is currently Full Professor of Optoelectronics at the University of Roma "Tor Vergata". His main field of activity is the theoretical study and numerical simulation of semiconductor nanostructures and devices. 

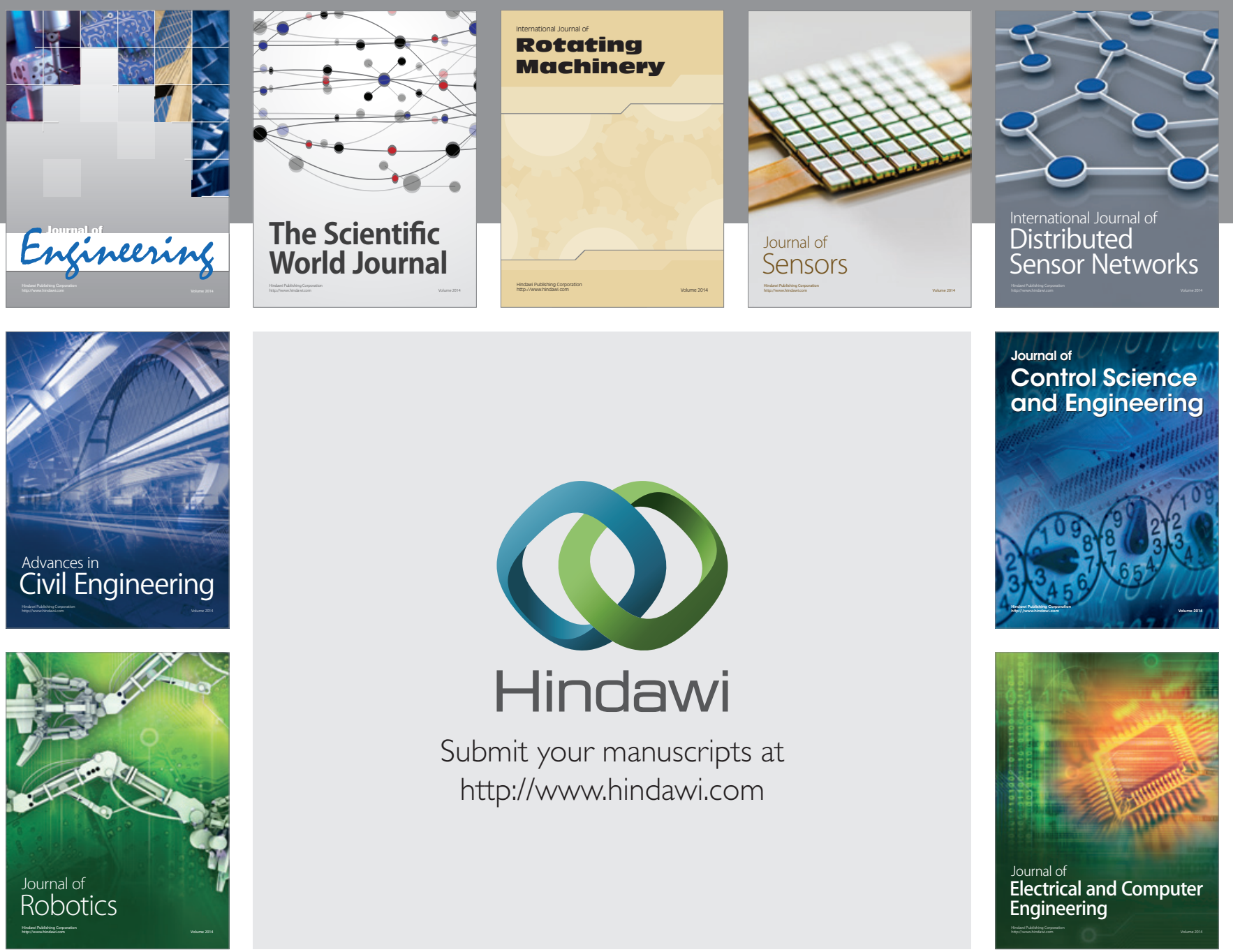

Submit your manuscripts at

http://www.hindawi.com
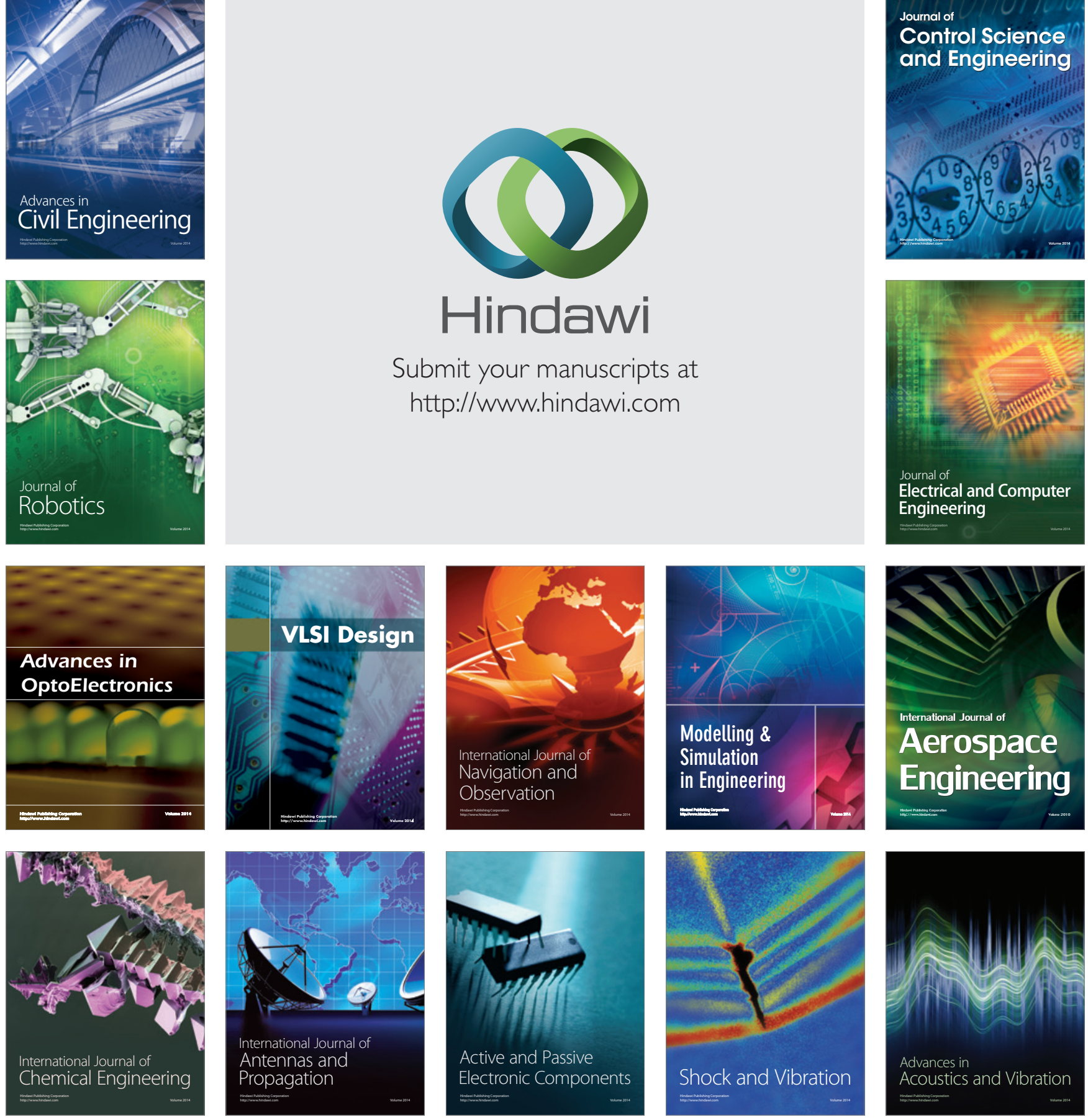